\title{
Bloqueo Para-Cervical Durante el Trabajo de Parto
}

\author{
Dr. Mario Mutis M. Instituto Colombiano de los \\ Seguros Sociales, Bucaramanga
}

\section{CAPITULO I}

\section{Introducción}

En el año 1926 se publica el primer trabajo de anestesia paracervical realizado por Gellert (1). Después de 20 años en 1945 Rosenfeld (2) publica en $E E$. UU . el primer trabajo en ese pais. pero hasta finales de la década del 50 y comienzos del 60 no se hace popular y se conoce con mayor amplitud.

La técnica ha sido la misma preconizada desde el primer momento con muy pequeñas variaciones: se coloca la paciente en posición ginecológica y se aplica en fondos vaginales laterales a la hora 4 y 8 de la esfera de un reloj. la cantidad de anestesia deseada. El líquido anestésico se inyecta en la base del ligamento ancho, de donde se extiende por el plexo útero-vaginal para bloquear las vías aferentes simpáticas y parasimpáticas al sistema nervioso central.

Se han usado varias agujas para esta técnica siendo las más comunes las de lowa-Trompet y la de Kobak.

La anestesia a usar ha sido aplicada en dosis única total o dosis de repetición, dejando un catéter permanente de Teflon como lo describieron Taffeen y colaboradores (4). Baggish en 1964 aplica una cánula de Teflón con este mismo fin e iguales resultados (3).
Se han usado distintos tipos de anestésicos siendo por lo tanto diferente su duración; fue así como Yates (5) recomendó la Lignocaína al $1 \%$ con adrenalina al 1:200.000 10 cc. a cada lado, obteniendo una duración promedio de 88 minutos. Cooper y Cols (6) en 1968 recomendaron la Bupivacaina al $0.5 \%$ con adrenalina al 1:200.000, obteniendo una duración promedio de 144 minutos.

Después de la inyección diferentes investigadores han observado bradicardia fetal, que está en proporción directa con la cantidad de droga anestésica empleada; se ha descrito una muerte fetal en útero y un caso después del nacimiento. Sterm y Col (7) (1969). Picton (8) en 100 casos reportó bradicardia fetal en el $6 \%$ y una muerte fetal a causa anestésica; Klas (9) en un estudio de 285 casos, no reportó bradicardia fetal usando Bupivacaína al $0.25 \%$ con epinefrina al 1:400.000. Al principio se creía que era la acción de la adrenalina sobre el feto, pero estudios posteriores han sugerido que es la acción del agente anestésico sobre el miocardio fetal. Shnider (10) y Col. 1968

\section{CAPITULO II}

\section{Material y métodos}

El presente estudio se hizo a pacientes del servicio de Obstetricia del ISS 
y del Hospital Universitario Ramón González Valencia de Bucaramanga sobre un total de 50 casos.

La técnica usada fue la preconizada anteriormente, colocando la paciente en posición ginecológica, con una aguja de punción lumbar calibre 22, cubierta por un tubo de polietileno $0.7 \mathrm{cc}$. más corto que la aguja para que sirviera de guía, permitiendo en esta forma introducir dentro de los tejidos el extremo distal de la aguja (0.7 c.c.) suficiente para inyectar la droga anestésica.

Con los dedos índice y medio de la mano izquierda se introduce en la vagina y sirve como guía para dirigir la aguja hacia los fondos vaginales derecho e izquierdo, y lograr colocar la misma a la hora 4 y 8 de la esfera del reloj donde se inyecta el líquido anestésico.

La droga que se eligió para el presente trabajo fue la Bupivacaína (Marcaína) por tratarse de una droga anestésica de larga duración.

La droga pertenece al grupo de las Xylidinas sintetizada por los laboratorios Bofers de Suecia por Ekenstan (11) y cuya diferencia química con la Mepivacaína radica en la sustitución del grupo metilo por el grupo butilo. Comercialmente se encuentra en solución salina al $0.5 \%$ con adrenalina al 1:200.000 y cuyo $\mathrm{Ph}$ es de 3.5 y en cuanto a su potencia anestésica es superior a la Mepivacaína y algo más tóxica que la misma (12).

La acción sobre el sistema cardiovascular estudiado por Jorfeld y Col (13) que inyectaron la droga por vía endovenosa en el hombre y el perro. Los resultados fueron: disminución del rendimiento cardíaco y volumen sistólico en un $20 \%$ y pequeño aumento de la resistencia periférica. La tensión arterial, la tensión venosa y la frecuencia cardíaca no registraron modificaciones. El O: arterial no se alteró y el equilibrio ácido/base sufrió una desviación hacia la acidosis metabólica.
En la sangre no presentó efecto sobre la eritropoyesis, lo mismo que no presentó acción hepatotóxica de acuer.do con los estudios hechos con bloqueos anestésicos durante 3 días haciendo exámenes de la Transaminasa Glutámico oxal-acética y Glutámico Pirúvica (14).

El efecto de la Bupivacaina sobre la transmisión del impulso nervioso fue estudiada electroneurográficamente durante varias semanas, después del bloqueo nervioso, no observándose daño ni secuela alguna $(15,16)$.

El valor primordial de esta droga radica en su larga duración de acción. La analgesia en bloqueos regionales epidulares alcanza a 8 hs. (17), en anestesia caudales 8.30 hs. (18), en bloqueos intercostales 16 hs., y en bloqueos paracervicales 144 minutos, Cooper (6).

En las series de estudios de diferentes autores consultores no se relatan complicaciones serias debido a la droga en sí, sino por error de técnicos.

\section{CAPITULO III}

\section{ANALISIS}

\section{Generalidades}

\section{Motivo}

El motivo de este estudio fue facilitar el trabajo de parto, durante el primer período, a las pacientes especialmente primigestantes, disminuyendo el dolor en las contracciones uterinas, la angustia y acortando el mismo.

Se toman como parámetros:

Edad: Los límites de edad fueron de 16 a 34 años así:

16 a 2.0 años $=15:=30 \%$

20 a 25 años $=25=50 \%$

25 a 30 años $=7=14 \%$

+ de 30 años $=3=6 \%$ 
Paridad: El mayor porcentaje fue de pacientes primigestantes por tratarse de personas que más lo necesitaban por su estado emocional y para tratar que el estudio se ajustara más a la realidad en cuanto al acortamiento del trabajo de parto.

$\mathrm{G} 1=37=74 \%$

$\mathrm{G} 2=11=22 \%$

$\mathrm{G} 3:=2=4 \%$

Edad Gestacional: La edad gestacional límite fue de las 32 a las 42 semanas siendo el mayor número de $40 \mathrm{se}$ manas.

32 semanas $=1=2 \%$
38 semanas $=1=2 \%$
39 semanas $=8=16 \%$
40 semanas $=25=50 \%$
41 semanas $=11=22 \%$
42 semanas $=4=8 \%$

Dosis Anestésica: La dosis total usada para cada paciente se inyectó en una sola aplicación, en ningún caso se repitió el bloqueo, ni se aplicó catéter para hacerla continua. Se usa distinta cantidad de droga a dosis iguales para cada lado, así:

\begin{tabular}{rccc}
\hline Dosis Total & Pacientes & Duración & Promedio \\
\hline $8 \mathrm{cC}$ & 6 & $101^{\prime}$ & \\
$10 \mathrm{cC}$ & 14 & $143^{\prime}$ & \\
$12 \mathrm{CC}$ & 17 & $146^{\prime}$ & $136^{\prime}$ \\
$14 \mathrm{cC}$ & 13 & $157^{\prime}$ & \\
\hline
\end{tabular}

De acuerdo con el cuadro adjunto observamos que el tiempo de duración está en proporción directa a la dosis. El promedio para la dosis de $14 \mathrm{cc}$ fue de 157 minutos, muy similar al trabajo realizado por Cooper y $\mathrm{Col}$ (6)

En el trabajo de Picton ( 8 ) con dosis de $20 \mathrm{cc}$. la duración fue de 258'. Dosis recomendada para este tipo de trabajo por no haber toxicidad y por la duración de su acción.

Se aclara que el promedio de duración puede ser mayor si no se hubiese acelerado el trabajo de parto en varios casos, llegando en uno a efectuarse el parto a los $75^{\prime}$ de aplicada la droga.

A pesar de todo nos da un promedio total de 136' de duración anestésica.

Dilatación: La infiltración se realizó cuando el cuello se encontraba a $3 \mathrm{~cm}$ de dilatación y hasta 6 , así:

$3 \mathrm{~cm}=12=24 \%$

$4 \mathrm{~cm}=21=42 \%$

$5 \mathrm{~cm}=15=30 \%$

$6 \mathrm{~cm}=2=\%$

Borramiento: $80 \%$ de borramiento en 5 pacientes, $90 \%$ en 21 pacientes y el $100 \%$ en 24 pacientes.

Presentación: En el $100 \%$ de las pacientes la presentación era cefálica.

Estación: La presentación no debe encontrarse muy encajada para poder practicar el bloqueo, de lo contrario, la cabeza fetal dificulta las maniobras. En el presente trabajo se llegó a infiltrar estando la presentación en +1 .

$-1=8=16 \%$

$0=22=44 \%$

$+1=20=40 \%$

Membranas: Integras en 35 pacientes para un $70 \%$. Rotas en 15 pacientes para un $30 \%$

Frecuencia Cardíaca Fetal: Hubo un aumento de la frecuencia cardiaca fetal en 15 casos, es decir, el $30 \%$. Disminución de la misma en 11 casos para un $22 \%$; no se alteró en 24 casos, para un $48 \%$.

En todos los casos en que se alteró la frecuencia cardíaca fetal ésta no pasó de + $\mathrm{O}$ - de 10; sólo en 2 casos hubo taquicardias de 160 , que se regularizaron antes de las 2 hs sin preocupar al obstetra y presentando partos normales y fetos con Apgar de 9 y $10 / 10$.

Tensión Arterial: En cuanto a la tensión arterial materna no hubo mayores 
variaciones. Aumentó en 10 casos para un $20 \%$. Disminuyó en 10 casos para un $20 \%$ y no se modificó en 30 casos o sea el $60 \%$. Las modificaciones fueron de $10 \mathrm{~mm}$ de mercurio; en un caso que ingresó con $150 \times 100$ (pre-eclampsia) disminuyó a 120 × 80 a los 5' y a $110 \times 70$ a las 2 horas.

Apgar: En términos generales los fetos nacieron con Apgar alto y no hubo muertes fetales.

$10,10=35=70 \%$
$9 / 10=6=12 \%$
$8 / 10=7=14 \%$
$7 / 10=2=$.

Tipo de Parto: En 38 pacientes se efectuó parto espontáneo para un $76 \%$. En 11 casos se aplicó fórceps para un $22 \%$ y en sólo un caso se realizó cesárea a una paciente primigestante de 34 años con desproporción céfalo-pélvica.

Drogas: En 14 casos $(28 \%)$ se aplicó venoclisis con Pitocín.

En $5(10 \%)$ se aplicó venoclisis con syntocinon y en $4(8 \%)$ se le dio a la paciente Oxamino-oxitocina (sandopart). Se aclara que estas conducciones en el trabajo del parto se hicieron con el solo fin de acelerarlo y no porque se hubieren paralizado o disminuido en su frecuencia e intensidad las contracciones uterinas. Aprovechando la buena anestesia que nos proporcionó la infiltración para-cervical se decidió aplicar dicho refuerzo.

\section{CAPITULO IV}

\section{Resumen y conclusiones}

1. Se practicó infiltración para-cervical con Bupivacaína (Marcaína) al $0.5 \%$ y al $1: 200.000$ de adrenalina, a 50 pacientes en el servicio de obstetricia del Hospital Universitario Ramón González Valencia y del ISS.
2. La edad promedio fue de 21.6 años.

3. La paridad entre G 1 y G 3 .

4. No hubo ninguna complicación seria lo que demuestra la nobleza del método y la droga.

5. No se presentó ninguna muerte fetal y Apgar fue alto

6. El promedio del tiempo anestésico fue de 157' cuando se infiltraron 14 cc. de la droga, promedio éste que se puede aumentar al aplicar $20 \mathrm{cc}$, lo que demuestra una buena duración que justifica el método.

7. Es un método sencillo, barato y rápido que no requiere gran experiencia y que se puede realizar en cualquier lugar del país.

\section{Recomiendo:}

a) Usar dosis más altas, hasta $20 \mathrm{cc}$ de Bupivacaína.

b) Método para ser practicado tanto en hospitales como en zonas rurales de nuestro país que carecen de medios económicos suficientes, por ser de fácil aplicación, no necesitar personal especializado, tratarse de una droga barata y de fácil adquisición y favorecer gran número de pacientes, que por su estado emocional y orgánico la necesitan.

\section{PARA-CERVICAL BLOCK DURING DELIVERY}

\section{Summary and conclusions:}

Para-cervical infiltration with Bupivacain (Marcain) at $0.5 \%$ and at 1:200.000 of adrenaline was applied to 50 patients at the Obstetrics Department of Hospital Universitario Ramón González Valencia and of ISS.

The average age was 21.6 years. Parity was between $G 1$ and $G 3$. No serious complications arouse, which show the effectiveness of both the method and the drug. No fetal death was regis- 
tered and the Apgar was high. The anesthetic time average was $157^{\prime}$ when 14 c.c. of the drug were applied; however this average can be increased when applying 20 c.c., thus justifying the method with such durantion. It is a simple. cheap and rapid method which does not require any special skill and can be executed in any part of the country.

\section{Suggest:}

a) To use higher doses, up to 20 c.c. of Bupicain.

b) This method can be used both in hospitals and rural areas of our country which lack economic resources, since it is easy to apply, do not need skilled personnel, it is an un-expensive and easy-to-acquire drug and it favours a great number of patients who need it due to their emotional and organic conditions.

\section{Bibliografía}

2 ROSENFELD: S. S. Paracervical anesttesia for relief of labor pains. Am. J. Glst. y Gy. nec. 50: 527. 1945

3 BAGGISH, M. S. 1964. Continuous paracervical block. Amer. J. Glustet - Gynec 88 968.

4 TAFFEEN CH., FRIEDMAN, H. L. HARRIS $H$.: Cambined continuous paracervical and continued fedeudal neuve block anesthesia in labor. Am. J. Obstet Gynecol 100: 55. 1968.

5 YATES, M. J. (1969). Painspul Labour: Modern methods of management. Proc. roy. Soc. Med. 62, 183

6 COOPER K., GIBROY K., J. and HURRY, D. J. (1968). Paracervical nerve block in labour using Bupivacaine. J. Obstet. Gyne. col. Brit. Cwlth 75, 863.

7 STERM, L., OUTERBIDGE, E. W., and fancett, J. S. 1969. Paracervical Block in obs. tetrics. Lancet 2, 322 .
8 F. C. R. PICTON. Paracervical Nerve Block in Labour. The British Journel of Cli. nical Practice Volume $23 \mathrm{~N}$ 4. April 1939

$\theta$ KLAS-HENRY HOEKEGARD, M. D. Marcai. ne for paracervical anesttesia during labor American No. 2 Pages 278-279. 1969.

10 SHNIDER S. M., ASLING, J. H., MARGO. LIS, A. J., WAY, E. L. and WILKINSON G. R. 1968. High poetal Blood levels

11 EKEMTAN B. AF., Maendal. Breve Rasseg ne lulle caracteristicle delba marcaine. $\mathrm{Mi}$. nerva anestesiológica 1968, 34, $498-500$

12 HEN F. and BRATTSAND F., Some Pharcno. cological and Toxicological Proferties of a Weur long acting local analgesic lac 43 (Marcaine) in comparison with Mupivacai ne. Acta Anesth. Scand 2969, supl XXI. 9-30.

13 JORFELD, LAFSTRON B. PERNOW F. WAHREN J. and WIDMAN B. I. V. Toxicity of Lac 43 in Dog and man Evaluated by Pluj. sioloyicol Methods. Proc 111 world Congress of Anesthesia 1964. Sau Paulo.

14 NORDQWIST P. and DHIRMEK K. G., Blood and Liver function during local anaestesia with Marcaine. Acta Awerth. Scand 1366 supl XXII

15 LOFSTRON B. WEMBERG A. and WIDDEN. L. Late Distrubances in Nerve function af. ter Block with local anaesthetis agents and Electronemographie Study. Acta Awerth Scand 1969, 10, 111, 112.

16 LOFSTRON B. WEMBERG A and WIDDEN L., and Electronemographie Study of Late Effects of Nerve Block. Proc. 111 World Congress of anaesthesiology 1964. Sau Paulo

17 EKLOM D. E. and WIDMAN B. A., Compa. rins of the Proferties of Lac 43 Prilucaine and Mupivacaine in Extradinal anesthesia. Acta Awerth. Scand 1966. Supl. XXI. 35-43.

18 HERBRING B. G. A. Comparative Study of lac 43 Mupivacaine and Anoesth. Scand 1966. Supl XXi, 45-52. 